\title{
Anxiety, depression, and stress in Korean patients with chronic urticaria
}

\author{
Gil-Soon Choi ${ }^{1}$, Young-Hee Nam ${ }^{2}$, Chan-Sun Park ${ }^{3}$, Mi-Yeong Kim ${ }^{4}$, Eun-Jung Jo ${ }^{5}$, Hye-Kyung Park ${ }^{5}$, \\ and Hee-Kyoo Kim ${ }^{1}$
}

\begin{abstract}
${ }^{1}$ Department of Internal Medicine, Kosin University College of Medicine, Busan; ${ }^{2}$ Department of Internal Medicine, Dong-A University College of Medicine, Busan; ${ }^{3}$ Department of Internal Medicine, Inje University Haeundae Paik Hospital, Busan; ${ }^{4}$ Department of Internal Medicine, Inje University Busan Paik Hospital, Busan; ${ }^{5}$ Department of Internal Medicine, Pusan National University School of Medicine, Busan, Korea
\end{abstract}

Received: September 26, 2019 Revised : November 1, 2019 Accepted: November 26, 2019

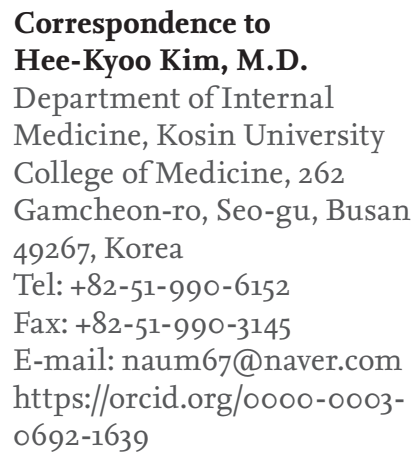

Background/Aims: Emotional distress is thought to cause or maintain chronic urticaria (CU). We aimed to investigate the presence of anxiety, depression, and stress in Korean adult CU patients and to explore their potential impact on treatment.

Methods: We enrolled $79 \mathrm{CU}$ patients and a disease control group comprising 39 persistent asthma patients. The Hospital Anxiety and Depression Scale (HADS) was used to evaluate depression and anxiety. Stress and quality of life (QoL) were assessed by Stress Response Inventory and CU-QoL questionnaires. The sociodemographic and clinical data such as urticaria activity score (UAS-15, UAS-6) were obtained.

Results: The prevalence of depression and anxiety based on the HADS were $48.1 \%$ and $38.0 \%$. Although the prevalence of anxiety didn't differ between the CU and asthma patients, depression was significantly more prevalent in the CU patients (48.1\% vs. $28.2 \%$ ). Stress tended to be lower in CU patients. Anxiety, depression, and stress exhibited negative correlations with QoL. Anxiety showed significant correlation with UAS-6 and pruritus-visual analog scale (VAS; $r=0.256, r=0.272$, $p<0.05$, respectively); depression correlated with sleep difficulty-VAS $(r=0.261, p<$ 0.05). Stress was associated with UAS-15, UAS-6, pruritus-VAS, and sleep difficulty-VAS $(r=0.251, r=0.317, r=0.302, r=0.258, p<0.05$, respectively).

Conclusions: The current study first presented that Korean CU patients frequently have anxiety and depression, which affect their QoL and demonstrated that anxiety, depression, and stress had different effects on sleep difficulty, pruritus, and urticaria severity in Korean CU patients.

Keywords: Chronic urticaria; Psychiatric disorders; Depression; Anxiety; Stress

\section{INTRODUCTION}

Chronic urticaria (CU) is defined by itchy wheals, with or without angioedema, that persist for more than 6 weeks [1]. It is a common skin disease with an estimated lifetime prevalence between $0.05 \%$ and 3\%. A nationwide epidemiological study using Korean health insurance data reported a remarkable increase in the prevalence of
CU between 2010 and 2014 [2].

Acute urticaria typically resolves quickly due to avoidance of the offending factors and symptomatic treatment. However, the underlying cause of CU is not identified in approximately $70 \%$ of patients, which makes management of the disease difficult and leads to frustration in both the patient and physician. Although few studies have evaluated the duration of CU to date, it is known 
to persist for 1 year in $70 \%$ of patients and can last for more than 5 years in up to $14 \%$ of patients $[3,4]$. A recent analysis reported a 5-year remission rate of CU in the Korean adult population to be $44.6 \%$ [5], demonstrating the long-term impact of CU. Moreover, CU has been reported to substantially impact the quality of life (QoL) of patients. Given that $\mathrm{CU}$ is a chronic and distressing illness, patients are likely to experience psychological distress. Indeed, a number of studies have shown that CU patients frequently exhibit psychiatric comorbidities [6-10]. Depression and anxiety are the most common psychiatric diagnoses found in CU patients. However, whether these psychiatric conditions predispose the patients to developing CU or emerge during the course of the disease is unclear. Nevertheless, psychological problems may contribute to the poor QoL reported by CU patients, as well as influence the outcome of urticaria treatments.

Although the management of CU is typically symptom-focused, it is also necessary to understand and address psychiatric conditions in patients. However, to the best of our knowledge, few studies have investigated psychiatric conditions in Korean CU patients. It is well known that cultural factors play a major role in psychiatric epidemiology [11]. Therefore, Korean CU patients may exhibit different psychiatric conditions compared to patients from other countries. We aimed to investigate the presence of anxiety, depression, and stress in Korean CU patients and to compare the prevalence of these psychiatric conditions in patients with bronchial asthma, as well as to explore the potential impact of psychiatric comorbidities on CU treatment.

\section{METHODS}

\section{Subjects}

A total of 79 patients with CU were prospectively recruited from five allergy centers. The inclusion criteria were: patients aged $\geq 19$ years and those who had urticaria symptoms for more than 6 weeks. Patients with urticarial vasculitis or other chronic skin diseases were excluded. Individuals with a diagnosis of a chronic or severe illness affecting QoL were also excluded. Thirty-nine patients with persistent asthma who were in partly- or uncontrolled asthmatic status while on the 2016 Global Initiative for Asthma step 3 or 4 during treatment were included in the study as disease controls. Patients were approached during their standard clinic visit and informed of the study. If they agreed to participate, they were enrolled. This study was approved by the Institutional Review Board of Kosin University Gospel Hospital (No. 2015-07-004), Dong-A University Hospital (No. 15118), Inje University Haeundae Paik Hospital (No. 201606-028), Inje University Busan Paik Hospital (No. 16-0133), and Pusan National University Hospital (No. H-1507-023032). Written informed consent was obtained from each patient.

\section{Sociodemographic and clinical data}

The sociodemographic and clinical data were obtained from the patients through a self-reported form that was developed by the investigators. Furthermore, the medical history of each patient, including disease duration, treatment step, and comorbidities, were verified by medical chart review.

All CU patients underwent an assessment of QoL and CU disease activity. QoL was assessed using a CU-QoL questionnaire developed and validated for measuring QoL in Korean CU patients [12]. This questionnaire is comprised of 17 items, and each is scored from 0 to 4 points, which results in a total score ranging from o to 68. A higher total score indicates a better QoL.

CU disease activity was assessed using the 15-point urticaria activity score (UAS-15), which measures five parameters based on the responder's experience over the preceding week: the number, distribution, duration, and diameter of wheals, and pruritus severity $[12,13]$. Each item is scored from o to 3, with the maximum UAS-15 being 15. A higher UAS-15 indicates more active urticaria. The 6-point UAS (UAS-6), which comprises the sum of the number of wheals and pruritus intensity, was also used [14]. We assessed the severity of pruritus and sleep difficulty symptoms using visual analog scales (VASs), as well.

\section{Assessment of anxiety, depression, and stress level} Anxiety and depression were assessed using the Hospital Anxiety and Depression Scale (HADS), which is the screening tool most commonly used by doctors to detect clinically significant states of anxiety and depression in non-psychiatric hospital settings [15]. The HADS consists of 14 items ( 7 items assessing anxiety and seven items assessing depression) with each item scored from 
1 to 3 points. The total HADS score ranges from o to 21 . A score $\geq 8$ is considered the standard cut-off for ascertaining anxiety or depression.

Stress levels were assessed using the Stress Response Inventory (SRI) questionnaire, which has been developed and validated for use in Korean populations [16]. The SRI measures reactions to emotional, physical, cognitive, and behavioral stress. It consists of 39 response items, each scored from o to 4 points, and the total SRI score ranging from o to 156 . A higher total score indicates higher stress levels.

\section{Statistical analysis}

Data are presented as mean \pm standard deviation or frequency (\%). In the bivariate analysis, the chi-square test and Student's $t$ test were used to compare categorical and continuous variables, respectively. Pearson's correlation tests were used to evaluate the associations be- tween psychological problems and urticaria status. A $p<$ 0.05 was considered statistically significant. All analyses were performed using the IBM SPSS, software version 24.0 (IBM Corp., Armonk, NY, USA).

\section{RESULTS}

\section{Clinical characteristics of study subjects}

A total of 79 Korean CU patients were enrolled in this study; the baseline characteristics of the participants are summarized in Table 1. The participants included 33 men (41.8\%) and 46 women (58.2\%) with a mean age of $37.5 \pm 14.8$ years. The average CU duration was $26.8 \pm 37.4$ months. CU was accompanied by angioedema, physical urticaria, and aspirin hypersensitivity in 14 (17.7\%), 19 (24.1\%), and five (6.3\%) patients, respectively. The mean UAS-15 and UAS-6 scores in CU patients were $7.6 \pm 3.4$

Table 1. Clinical characteristics of the study subjects

\begin{tabular}{|c|c|c|}
\hline Variable & Chronic urticaria $(\mathrm{n}=79)$ & Bronchial asthma $(\mathrm{n}=39)$ \\
\hline Age, yr & $37.5 \pm 14.8(18-74)$ & $52.8 \pm 15.0(18-88)$ \\
\hline Male sex & $33(41.8)$ & $16(41.0)$ \\
\hline Alcohol, current & $43(54 \cdot 4)$ & NA \\
\hline Atopy & $31(39.2)$ & NA \\
\hline Serum total IgE, kU/L & $316.4 \pm 340.9$ & NA \\
\hline Disease duration, mon & $26.8 \pm 37.4$ & NA \\
\hline Treatment duration, mon & $17.3 \pm 32.6$ & NA \\
\hline Angioedema & $14(17.7)$ & NA \\
\hline Physical urticaria & $19(24.1)$ & NA \\
\hline Aspirin hypersensitivity & $5(6.3)$ & NA \\
\hline CU-QoL & $39 \cdot 5 \pm 15.1$ & NA \\
\hline UAS-15/UAS-6 & $7 \cdot 6 \pm 3 \cdot 4 / 3.2 \pm 1.6$ & NA \\
\hline Pruritus/sleep difficulty VAS score & $4 \cdot 6 \pm 3 \cdot 3 / 3 \cdot 4 \pm 3 \cdot 4$ & NA \\
\hline Treatment step & & NA \\
\hline No medication & $1(1.3)$ & \\
\hline$\leq 2$ Antihistamines & $43(54 \cdot 4)$ & \\
\hline 3-4 Antihistamines & $21(26.6)$ & \\
\hline With LTRA & $4(5.1)$ & \\
\hline With omalizumab & $8(10.1)$ & \\
\hline With cyclosporine \pm steroid & $2(2.5)$ & \\
\hline
\end{tabular}

Values are presented as mean $\pm \mathrm{SD}$ (range), number (\%), or mean $\pm \mathrm{SD}$.

NA, not available; IgE, immunoglobulin E; CU-QoL, chronic urticaria-specific quality of life; UAS, urticaria activity score; VAS, visual analog scale; LTRA, leukotriene receptor antagonist. 
Table 2. Anxiety, depression, and stress levels in chronic urticaria and bronchial asthma patients

\begin{tabular}{lccc}
\hline Variable & Chronic urticarial $(\mathrm{n}=79)$ & Bronchial asthma $(\mathrm{n}=39)$ & $p$ value \\
\hline Anxiety & $30(38.0)$ & $16(41.0)$ & 0.451 \\
Depression & $38(48.1)$ & $11(28.2)$ & 0.039 \\
HADS total score & $12.9 \pm 6.5$ & $12.4 \pm 8.0$ & 0.723 \\
HADS anxiety score & $5.8 \pm 3.6$ & $7.1 \pm 4.5$ & 0.121 \\
HADS depression score & $7.2 \pm 3.6$ & $5.3 \pm 3.9$ & 0.012 \\
Stress level & $26.6 \pm 26.3$ & $36.9 \pm 27.4$ & 0.057 \\
\hline
\end{tabular}

Values are presented as number (\%) or mean \pm SD.

A HADS score $\geq 8$ is considered the standard cut-off for the indication of anxiety or depression. Stress levels were assessed using the Stress Response Inventory questionnaire. Statistical significance was evaluated by $t$ tests and chi-square tests.

HADS, Hospital Anxiety and Depression Scale.

Table 3. Correlation between anxiety/depression/stress level and urticaria status

\begin{tabular}{lccc}
\hline Parameter & Anxiety score & Depression score & Stress level \\
\hline Anxiety score & - & $0.609^{\mathrm{a}}$ & $0.736^{\mathrm{a}}$ \\
Depression score & $0.609^{\mathrm{a}}$ & - & $0.545^{\mathrm{a}}$ \\
CU-QoL & $-0.454^{\mathrm{a}}$ & $-0.232^{\mathrm{b}}$ & $-0.587^{\mathrm{a}}$ \\
UAS-15 & 0.222 & 0.148 & $0.251^{\mathrm{c}}$ \\
UAS-6 & $0.256^{\mathrm{c}}$ & 0.186 & $0.317^{\mathrm{b}}$ \\
Pruritus VAS score & $0.272^{\mathrm{c}}$ & 0.190 & $0.302^{\mathrm{b}}$ \\
Sleep difficulty VAS score & $0.205^{\mathrm{c}}$ & $0.249^{\mathrm{c}}$ & $0.25^{\mathrm{c}}$ \\
Treatment step & -0.149 & -0.182 & -0.123 \\
\hline
\end{tabular}

Statistical significance was evaluated by Pearson's correlation test.

CU-QoL, chronic urticaria-specific quality of life; UAS, urticaria activity score; VAS, visual analog scale.

${ }^{\mathrm{a}} p<0.001$.

${ }^{\mathrm{b}} \mathrm{p}<0.01$.

${ }^{\mathrm{c}} p<0.05$.

and $3.2 \pm 1.6$, respectively.

\section{Anxiety, depression, and stress levels in the study subjects}

As shown in Table 2, the prevalence of anxiety and depression in the study and control groups was determined and compared. Additionally, the stress level was also assessed in these two groups. The mean HADS score in CU patients was $12.9 \pm 6.5$. Based on the HADS, the prevalence of depression and anxiety in CU patients was $48.1 \%$ and $38.0 \%$, respectively. The prevalence of anxiety did not significantly differ between the CU and asthma patients (38.0\% vs. 41.0\%). However, depression was significantly more prevalent in CU patients $(48.1 \%$ vs. $28.2 \%, p<0.039$ ). While stress levels tended to be higher in asthmatic participants, the difference was not statistically significant.

\section{Correlation between urticaria status and anxiety, depression, and stress levels}

To investigate the effects of psychological problems on $\mathrm{CU}$, we evaluated the associations between urticaria status and anxiety, depression, and stress levels (Table 3).

Anxiety, depression, and stress levels were significantly correlated with each other $(p<0.001$ for all comparisons) and exhibited significant negative correlations with QoL (anxiety: $r=-0.454, p<0.001$; depression: $r=$ $-0.232, p<0.05$; stress $r=-0.587, p<0.001$, respectively). No significant associations were observed between these psychological conditions and the current treatment 
step. Anxiety scores were significantly correlated with UAS-6 scores and pruritus VAS scores $(r=0.256, p<0.05$ and $r=0.272, p<0.05$, respectively) but was not correlated with sleep difficulty VAS scores. No significant correlations were observed between depression scores and UAS (UAS-15 or UAS-6) or pruritus VAS scores. Depression scores were significantly correlated with sleep difficulty VAS scores $(r=0.261, p<0.05)$. Stress levels were significantly correlated with UAS-15, UAS-6, and pruritus VAS scores, as well as sleep difficulty VAS scores $(r=0.251, p<$ $0.05 ; r=0.317, p<0.01 ; r=0.302, p<0.01 ;$ and $r=0.258, p<$ 0.05 , respectively).

\section{Determinants of anxiety and depression in patients with CU}

Table 4 shows the clinical characteristics of CU patients with anxiety/depression. Compared to CU patients without anxiety, CU patients with anxiety exhibited lower CU-QoL scores (37.2 \pm 13.7 vs. $50.6 \pm 13.6, p<0.001)$, higher pruritus VAS scores (5.8 \pm 3.1 vs. $3.9 \pm 3.2, p<0.05)$, higher UAS-6 scores ( $3.7 \pm 1.5$ vs. $2.9 \pm 1.6, p<0.05)$, and higher stress levels $(49.3 \pm 26.7$ vs. $12.9 \pm 13.4, p<0.001)$. Compared to CU patients without depression, CU patients with depression exhibited lower log-transformed serum total immunoglobulin E (IgE) levels and higher stress levels ( $p<0.05$ and $p<0.001$, respectively). CU-QoL scores tended to be lower in subjects with depression $(p=0.063)$. No significant differences in gender, smoking history, comorbidities, disease duration, or current treatment steps were observed between CU patients with and without anxiety/depression.

\section{DISCUSSION}

Psychiatric factors are thought to be involved in the development and aggravation of CU. However, little information is available with respect to anxiety, depression, and stress in Korean CU patients. In the present study, we evaluated these conditions in CU patients using the HADS and SRI questionnaires. We confirmed that CU patients frequently presented with psychiatric conditions and observed a high prevalence (48.1\% and $38.0 \%)$ of depression and anxiety in these individuals. Our study is the first investigation to evaluate the prevalence of anxiety and depression in Korean CU patients. These results are similar to those of a previous meta-analysis that evaluated the prevalence of anxiety and depression in CU patients and reported a pooled prevalence of $46.09 \%$ [17]. In Korea, it was recently reported that the prevalence of depression in patients with urticaria was similar to that in patients with atopic dermatitis $30 \%$ in adults), while the prevalence of anxiety was lower in atopic dermatitis ( $43 \%$ in adults) in an analysis using the Korean National Health Insurance Research Database [18]. These results were different from ours, which may be due to differences in the urticaria patients enrolled in the study. The referenced study included patients defined as urticaria (L50.9 by the International Classification of Disease [ICD]-10), which included a wide range of urticaria, as well as CU (L50.8 by ICD-10). Previous studies have reported that patients with CU were more depressed and anxious than healthy controls, although a number of other studies failed to corroborate that result $[19,20]$. In this study, we compared the prevalence of psychological problems between CU patients and asthma patients. Asthma was employed as a control condition in this study, as it is a common chronic allergic disease with a relatively well known impact of psychiatric comorbidities [21-25]. We found that depression was significantly more prevalent in CU patients compared to patients with asthma (48.1\% vs. $28.2 \%, p<0.039)$. In contrast, the prevalence of anxiety was similar between the CU and asthma patients, with CU patients exhibiting a lower stress level. These results suggest that the presence of psychological comorbidities, especially depression, should be considered in the management of CU.

The role of depression, anxiety, and stress in CU remains unclear. Some studies have suggested that psychiatric problems play a role in the etiology or exacerbation of urticaria [8,26-28]. Meanwhile, others have reported that dermatological diseases are risk factors for psychiatric disorders, such as depression and anxiety [7]. While it is not possible to determine the causal relationship between psychological problems and CU, it is thought that psychological problems are related to QoL and disease control. Indeed, previous studies have reported that psychiatric comorbidities were associated with a more pronounced reduction of QoL in CU patients $[6,8,29,30]$. Consistent with previous studies, we identified significant negative correlations between QoL and depression scores, anxiety scores, and stress levels, which suggests 
Table 4. Characteristics of patients with chronic urticaria with and without anxiety/depression

\begin{tabular}{|c|c|c|c|c|}
\hline Parameter & Anxiety $(\mathrm{n}=30)$ & No anxiety $(\mathrm{n}=49)$ & Depression $(n=38)$ & No depression $(n=41)$ \\
\hline Age, yr & $35 \cdot 3 \pm 11.2$ & $38.9 \pm 15.7$ & $37.9 \pm 12.3$ & $37.2 \pm 15 \cdot 9$ \\
\hline Male sex & $9(30.0)$ & $24(49.0)$ & $(31.6)$ & $21(51.2)$ \\
\hline Disease duration, mon & $23.2 \pm 29.1$ & $29.1 \pm 42.0$ & $24.8 \pm 29 \cdot 3$ & $28.7 \pm 44.3$ \\
\hline Treatment duration, mon & $15.8 \pm 21.2$ & $18.2 \pm 38.2$ & $17.2 \pm 24.4$ & $17.5 \pm 39.2$ \\
\hline Atopy & $7(23 \cdot 3)$ & $24(49.0)$ & $12(31.6)$ & $19(46.3)$ \\
\hline Ln (total IgE), kU/L & $5.1 \pm 0.8$ & $5 \cdot 4 \pm 1.0$ & $4.9 \pm 0.9^{a}$ & $5.6 \pm 1.0$ \\
\hline Angioedema & $6(20.0)$ & $8(16.3)$ & $4(10.5)$ & $10(24 \cdot 4)$ \\
\hline Living with family & $25(83.3)$ & $33(67 \cdot 3)$ & $30(78.9)$ & $28(68.3)$ \\
\hline Job-status, currently working & $21(70)$ & $29(59.1)$ & $22(57 \cdot 9)$ & $28(68.3)$ \\
\hline \multicolumn{5}{|l|}{ Economic status (won) } \\
\hline$<1,500,000$ & $5(16.7)$ & $6(12.2)$ & $6(15.8)$ & $5(12.2)$ \\
\hline $1,500,000-4,000,000$ & $16(53 \cdot 3)$ & $27(55 \cdot 1)$ & $23(60.5)$ & $20(48.8)$ \\
\hline$>4,000,000$ & $9(30)$ & $16(32.7)$ & $9(23.7)$ & $16(39.0)$ \\
\hline \multicolumn{5}{|l|}{ Education } \\
\hline Middle school or below & $1(3 \cdot 3)$ & $6(12.2)$ & $3(7 \cdot 9)$ & $4(9.8)$ \\
\hline High school & $17(56.7)$ & $27(55.1)$ & $21(55 \cdot 3)$ & $23(56.1)$ \\
\hline College or above & $12(40.0)$ & $16(32.6)$ & $14(36.8)$ & $14(34.1)$ \\
\hline \multicolumn{5}{|l|}{ Treatment step } \\
\hline No medication & $1(3 \cdot 3)$ & o & o & $1(2.4)$ \\
\hline$\leq 2$ Antihistamines & $17(56.7)$ & $26(53.1)$ & $24(63.2)$ & $19(46.3)$ \\
\hline 3-4 Antihistamines & $8(26.7)$ & $13(26.5)$ & $8(21.1)$ & $13(31.7)$ \\
\hline With LTRA & $1(3 \cdot 3)$ & $3(6.1)$ & $1(2.6)$ & $3(7 \cdot 3)$ \\
\hline With omalizumab & $3(10.0)$ & $5(10.2)$ & $4(10.5)$ & $4(9.8)$ \\
\hline With cyclosporine \pm steroid & 0 & $2(4 \cdot 1)$ & $1(2.6)$ & $1(2.4)$ \\
\hline UAS-15 & $8.5 \pm 3.1$ & $7 \cdot 0 \pm 3 \cdot 5$ & $7.5 \pm 0.3$ & $7 \cdot 7 \pm 3 \cdot 5$ \\
\hline UAS-6 & $3.7 \pm 1.5^{\mathrm{c}}$ & $2.9 \pm 1.6$ & $3 \cdot 3 \pm 1.5$ & $3.2 \pm 1.7$ \\
\hline Pruritus VAS score & $5.8 \pm 3.1^{\mathrm{c}}$ & $3.9 \pm 3.2$ & $4.9 \pm 3.1$ & $4 \cdot 4 \pm 3 \cdot 4$ \\
\hline Sleep difficulty VAS score & $4.3 \pm 3.8$ & $2.8 \pm 3.0$ & $3.7 \pm 3.6$ & $3.0 \pm 3.2$ \\
\hline CU-QoL & $37.2 \pm 13.7^{\mathrm{d}}$ & $50.6 \pm 13.6$ & $42.2 \pm 13.9$ & $48.5 \pm 15 \cdot 7$ \\
\hline Stress level & $49 \cdot 3 \pm 26.7^{d}$ & $12.9 \pm 13.4$ & $42.5 \pm 28.0^{b}$ & $12.0 \pm 12.7$ \\
\hline
\end{tabular}

Values are presented as mean \pm SD or number (\%). Statistical significance was evaluated by $t$ tests and chi-square tests.

IgE, immunoglobulin E; LTRA, leukotriene receptor antagonist; UAS, urticaria activity score; VAS, visual analog scale; CUQoL, chronic urticaria-specific quality of life.

${ }^{\mathrm{a}} p<0.05,{ }^{b} p<0.001$ (depression group vs. no depression group).

${ }^{c} p<0.05,{ }^{d} p<0.001$ (anxiety group vs. no anxiety group).

that psychiatric conditions greatly impact QoL.

Psychological conditions may relate to the disease status of CU. In this study, we investigated disease activity using UAS scores, instead of assessing the urticaria control status (described using categories well-, partly-, and uncontrolled). Another important finding of this study that has rarely been noted in previous research is that anxiety, depression, and stress impact CU differently. Gupta et al. [31] reported a direct correlation between pruritus severity and depression score in patients with 
pruritic skin disorders, including CU. However, this association was not identified in another study. Recent studies have reported that depression scores were not associated with UAS $[9,30,32]$, indicating the absence of a significant relationship between disease activity and depression. We also found that depression scores were not associated with UAS or pruritus severity in the present study but instead were associated with the severity of sleep difficulty. It is postulated that sleep difficulty could act as both an effect of depression and a risk factor for the development of depression [33,34]. Taking into account previously published results, our results suggest that sleep difficulties may be related to depression in CU patients. In this study, we found the anxiety score to be associated with UAS- 6 scores and pruritus severity. Anxiety can exacerbate itch, leading to a vicious cycle that affects patient behavior and worsens disease prognosis. Given that the UAS-6 is based on the number of wheals and pruritus severity, anxiety is very likely related to pruritus severity. Finally, previous studies have reported that stress levels correlate with CU disease activity and severity. Similarly, our results demonstrated that stress levels were significantly correlated with all parameters of UAS, severity of sleep difficulty, and pruritus severity, as well as depression and anxiety scores.

Various demographic and lifestyle factors have been reported to be risk factors for depression and anxiety in the general population. Our results demonstrate that higher stress levels are a risk factor for depression and anxiety among CU patients, regardless of demographic and lifestyle factors. It is well known that stress contributes to the development of depression and anxiety in other diseases [35,36], which is consistent with the results of our study. These findings indicate that stress levels can be used as a marker to predict the presence of depression and anxiety. Additionally, our study showed that anxiety was more commonly noted in subjects with a higher UAS-6 score, a higher pruritus VAS score, and a lower QoL, which suggests that these parameters may be considered as risk factors for anxiety.

The serum IgE levels of CU patients are increasingly becoming a focus of research due to the clinical benefits of anti-IgE treatment with omalizumab. Although the role of total IgE levels is still not well defined, there are reports stating that elevated total serum IgE level correlates with the severity of urticaria and QoL impair- ment $[13,37]$. However, another study did not show any relationship between total IgE levels and the severity of urticaria [38]. In this study, we did not find any significant correlation between total IgE levels and urticaria severity (data not shown). However, we did note a lower total IgE level in CU patients with depression. Recently, a lower response rate to omalizumab has been reported in CU patients with low levels of total IgE [39,40]. It is currently unclear why lower IgE levels are linked to depression. One explanation may be that poor response to treatment may be related to the depressive mood in CU patients.

The mechanism by which psychological conditions impact CU has not been elucidated. However, two pathological mechanisms have been suggested $[41,42]$. One potential theory is that $\mathrm{CU}$ results from interactions between the immune and nervous systems, in which mast cell activation is elicited through the hypothalamic-pituitary-adrenocortical axis, the sympathetic and adrenomedullary system, and local skin nerve fibers. The alternate theory is that $\mathrm{CU}$ occurs due to stress-related mechanisms. Psychological stress can induce the release of cortisol and the expression of inflammatory mediators, leading to the activation of cutaneous mast cells and the development of CU. As psychiatric conditions often accompany CU in patients, psychological interventions have been tried and have been reported to be effective in the treatment of CU [43-46]. However, there is limited research on these treatments, and studies are often performed in small patient groups. Therefore, further research is warranted.

There are several limitations to this study. First, depression and anxiety were assessed using a questionnaire and not by a physician. Although the HADS cannot fully replace a psychiatric interview, it is a commonly used and validated tool in the setting of non-psychiatric hospitals. Second, the cross-sectional design of the study prevents the determination of causal relationships. Finally, this study included a relatively small number of patients. In particular, fewer asthma patients were enrolled as disease controls, which may limit the interpretation of the observation that CU patients are more likely to exhibit depression than asthma patients (despite the statistically significant difference). Therefore, our data may be considered preliminary, which necessitates further studies to elaborate on our results. 
In conclusion, this study is the first to investigate the prevalence of depression and anxiety in Korean CU patients. Our data confirmed that CU patients are frequently affected by psychiatric conditions, particularly depression, which affects the QoL of Korean CU patients. We found that depression was associated with sleep difficulty; anxiety was associated with pruritus severity and urticarial activity; and stress was associated with sleep difficulty, pruritus severity, and urticarial activity. Overall, it is important to consider the potential existence of psychiatric conditions in the management of patients with CU.

\section{KEY MESSAGE}

1. This is the first study showing that Korean chronic urticaria (CU) patients frequently have psychiatric comorbidities, and we report a high prevalence of depression and anxiety $(48.1 \%$ and $38.0 \%$, respectively) in these individuals.

2. We demonstrated that depression is associated with sleep difficulties; anxiety is associated with pruritus severity and urticaria severity; and stress is associated with sleep difficulty, pruritus severity, and urticaria severity in Korean CU patients. Overall, it is important to consider the potential presence of psychiatric conditions in the management of patients with CU.

\section{Conflict of interest}

No potential conflict of interest relevant to this article was reported.

\section{Acknowledgments}

This study was supported by a grant from the Kosin University College of Medicine.

\section{REFERENCES}

1. Zuberbier T, Aberer W, Asero R, et al. The EAACI/GA(2) LEN/EDF/WAO guideline for the definition, classification, diagnosis, and management of urticaria: the 2013 revision and update. Allergy 2014;69:868-887.

2. Lee N, Lee JD, Lee HY, Kang DR, Ye YM. Epidemiology of chronic urticaria in Korea using the Korean Health Insurance Database, 2010-2014. Allergy Asthma Immunol Res 2017;9:438-445.

3. Gaig P, Olona M, Munoz Lejarazu D, et al. Epidemiology of urticaria in Spain. J Investig Allergol Clin Immunol 2004;14:214-220.

4. Ye YM, Park JW, Kim SH, et al. Prognostic factors for chronic spontaneous urticaria: a 6-month prospective observational study. Allergy Asthma Immunol Res 2016;8:115-123.

5. Kim YS, Park SH, Han K, et al. Clinical course of chronic spontaneous urticaria in the Korean adult population. Allergy Asthma Immunol Res 2018;10:83-87.

6. Ozkan M, Oflaz SB, Kocaman N, et al. Psychiatric morbidity and quality of life in patients with chronic idiopathic urticaria. Ann Allergy Asthma Immunol 2007;99:29-33.

7. Chung MC, Symons C, Gilliam J, Kaminski ER. The relationship between posttraumatic stress disorder, psychiatric comorbidity, and personality traits among patients with chronic idiopathic urticaria. Compr Psychiatry 2010;51:55-63.

8. Staubach P, Dechene M, Metz M, et al. High prevalence of mental disorders and emotional distress in patients with chronic spontaneous urticaria. Acta Derm Venereol 2011;91:557-561.

9. Herguner S, Kilic G, Karakoc S, Tamay Z, Tuzun U, Guler N. Levels of depression, anxiety and behavioural problems and frequency of psychiatric disorders in children with chronic idiopathic urticaria. Br J Dermatol 2011;164:1342-1347.

10. Maurer M, Dechene M, Staubach P. Two out of three patients with chronic idiopathic urticaria exhibit psychiatric comorbidity. Allergo J 2003;12:54.

11. Patel V. Cultural factors and international epidemiology. Br Med Bull 2001;57:33-45.

12. Ye YM, Park JW, Kim SH, et al. Clinical evaluation of the computerized chronic urticaria-specific quality of life questionnaire in Korean patients with chronic urticaria. Clin Exp Dermatol 2012;37:722-728.

13. Choi WS, Lim ES, Ban GY, et al. Disease-specific impairment of the quality of life in adult patients with chronic spontaneous urticaria. Korean J Intern Med 2018;33:185192.

14. Zuberbier T, Aberer W, Asero R, et al. Methods report on the development of the 2013 revision and update of the EAACI/GA2 LEN/EDF/WAO guideline for the definition, 
classification, diagnosis, and management of urticaria. Allergy 2014;69:e1-e29.

15. Zigmond AS, Snaith RP. The hospital anxiety and depression scale. Acta Psychiatr Scand 1983;67:361-370.

16. Koh KB, Park JK, Kim CH, Cho S. Development of the stress response inventory and its application in clinical practice. Psychosom Med 2001;63:668-678.

17. Ben-Shoshan M, Blinderman I, Raz A. Psychosocial factors and chronic spontaneous urticaria: a systematic review. Allergy 2013;68:131-141.

18. Ahn HJ, Shin MK, Seo JK, et al. Cross-sectional study of psychiatric comorbidities in patients with atopic dermatitis and nonatopic eczema, urticaria, and psoriasis. Neuropsychiatr Dis Treat 2019;15:1469-1478.

19. Topal IO, Kivanc Altunay I, Mercan S. Personality disorders, anxiety and depression in the patients with chronic urticaria. J Clin Psychiatry 2004;7:199-209.

20. Sheehan-Dare RA, Henderson MJ, Cotterill JA. Anxiety and depression in patients with chronic urticaria and generalized pruritus. Br J Dermatol 1990;123:769-774.

21. Kuehn BM. Asthma linked to psychiatric disorders. JAMA 2008;299:158-160.

22. Lavoie KL, Bacon SL, Barone S, Cartier A, Ditto B, Labrecque $M$. What is worse for asthma control and quality of life: depressive disorders, anxiety disorders, or both? Chest 2006;130:1039-1047.

23. Richardson LP, Russo JE, Lozano P, McCauley E, Katon W. The effect of comorbid anxiety and depressive disorders on health care utilization and costs among adolescents with asthma. Gen Hosp Psychiatry 2008;30:398-406.

24. Deshmukh VM, Toelle BG, Usherwood T, O'Grady B, Jenkins CR. The association of comorbid anxiety and depression with asthma-related quality of life and symptom perception in adults. Respirology 2008;13:695-702.

25. Ciprandi G, Schiavetti I, Rindone E, Ricciardolo FL. The impact of anxiety and depression on outpatients with asthma. Ann Allergy Asthma Immunol 2015;115:408-414.

26. Picardi A, Abeni D. Stressful life events and skin diseases: disentangling evidence from myth. Psychother Psychosom 2001;70:118-136.

27. Kimyai-Asadi A, Usman A. The role of psychological stress in skin disease. J Cutan Med Surg 2001;5:140-145.

28. Barbosa F, Freitas J, Barbosa A. Chronic idiopathic urticaria and anxiety symptoms. J Health Psychol 2011;16:1038-1047.

29. Staubach P, Eckhardt-Henn A, Dechene M, et al. Quality of life in patients with chronic urticaria is differentially impaired and determined by psychiatric comorbidity. $\mathrm{Br}$ J Dermatol 2006;154:294-298.

30. Engin B, Uguz F, Yilmaz E, Ozdemir M, Mevlitoglu I. The levels of depression, anxiety and quality of life in patients with chronic idiopathic urticaria. J Eur Acad Dermatol Venereol 2008;22:36-40.

31. Gupta MA, Gupta AK. Depression modulates pruritus perception: a study of pruritus in psoriasis, atopic dermatitis and chronic idiopathic urticaria. Ann N Y Acad Sci 1999;885:394-395.

32. Uguz F, Engin B, Yilmaz E. Axis I and Axis II diagnoses in patients with chronic idiopathic urticaria. J Psychosom Res 2008;64:225-229.

33. Breslau N, Roth T, Rosenthal L, Andreski P. Sleep disturbance and psychiatric disorders: a longitudinal epidemiological study of young adults. Biol Psychiatry 1996;39:411418.

34. Lustberg L, Reynolds CF. Depression and insomnia: questions of cause and effect. Sleep Med Rev 2000;4:253262.

35. Cohen BE, Edmondson D, Kronish IM. State of the art review: depression, stress, anxiety, and cardiovascular disease. Am J Hypertens 2015;28:1295-1302.

36. Bohlmeijer E, Prenger R, Taal E, Cuijpers P. The effects of mindfulness-based stress reduction therapy on mental health of adults with a chronic medical disease: a meta-analysis. J Psychosom Res 2010;68:539-544.

37. Kessel A, Helou W, Bamberger E, et al. Elevated serum total IgE: a potential marker for severe chronic urticaria. Int Arch Allergy Immunol 2010;153:288-293.

38. Toubi E, Kessel A, Avshovich N, et al. Clinical and laboratory parameters in predicting chronic urticaria duration: a prospective study of 139 patients. Allergy 2004;59:869873.

39. Straesser MD, Oliver E, Palacios T, et al. Serum IgE as an immunological marker to predict response to omalizum$\mathrm{ab}$ treatment in symptomatic chronic urticaria. J Allergy Clin Immunol Pract 2018;6:1386-1388.

40. Weller K, Ohanyan T, Hawro T, et al. Total IgE levels are linked to the response of chronic spontaneous urticaria patients to omalizumab. Allergy 2018;73:2406-2408.

41. Yang HY, Sun CC, Wu YC, Wang JD. Stress, insomnia, and chronic idiopathic urticaria: a case-control study. J Formos Med Assoc 2005;104:254-263.

42. Pasaoglu G, Bavbek S, Tugcu H, Abadoglu O, Misirligil Z. 
Psychological status of patients with chronic urticaria. J Dermatol 2006;33:765-771.

43. Shenefelt PD. Psychological interventions in the management of common skin conditions. Psychol Res Behav Manag 2010;3:51-63.

44. Tole JW, Gratz KL, Dave ND, Smith SK, Marshall GD. Effect of psychological stress intervention for patients with chronic urticaria (CU). J Allergy Clin Immunol
2011;127:AB104.

45. Sperber J, Shaw J, Bruce S. Psychological components and the role of adjunct interventions in chronic idiopathic urticaria. Psychother Psychosom 1989;51:135-141.

46. Schut C, Mollanazar NK, Kupfer J, Gieler U, Yosipovitch G. Psychological interventions in the treatment of chronic itch. Acta Derm Venereol 2016;96:157-161. 